\title{
FLOWERING HTH1 is involved in CONSTANS-mediated flowering regulation in Arabidopsis
}

\author{
Soon Ae Sim ${ }^{1,2}$, Su Gyeong Woo', Dae Yeon Hwang 3 , Jin-Hong Kim', Seung Sik Lee ${ }^{4,5}$, Chae Oh Lim', \\ Jong Chan Hong ${ }^{1,6^{*}}$ and Young Hun Song ${ }^{3^{*}}$ (1)
}

\begin{abstract}
Flowering at the right time is essential for maximum reproductive fitness. In Arabidopsis thaliana, the CONSTANS (CO) protein facilitates the transition from the vegetative phase to the reproductive phase under long-day conditions. The formation of heterodimeric complexes between CO and DNA binding domain-containing transcription factors is important for the induction of day length-dependent flowering. Here, we report a myb-like helix turn helix (HTH) transcriptional regulator family protein as a new modulator of floral transition, which we have named FLOWERING HTH1 (FHTH1). We isolated FHTH1 as a CO-interacting protein by a yeast two-hybrid screen using an Arabidopsis transcription factor library. Our analysis showed that FHTH1 presented in the nucleus and the FHTH1-CO complex was formed in the same subcellular location. We also observed the expression of a FHTH1:GUS construct in the leaf vasculature, where $\mathrm{CO}$ exists. Transgenic plants overexpressing FHTH1 fused with the plant-specific repression domain SRDX showed a delayed flowering phenotype in long days, resembling the phenotype of the co mutant. Our results suggest that $\mathrm{FHTH} 1$ may contribute to $\mathrm{CO}$-mediated photoperiodic flowering regulation.
\end{abstract}

Keywords: Flowering time, CONSTANS, Photoperiod, Myb-like helix turn helix transcriptional regulator, Chimeric repressor

\section{Introduction}

Plants sense seasonal changes and align their development with the surrounding environment to maximize reproductive fitness $[1,2]$. One of the most important environmental factors controlling the transition from the vegetative phase to the reproductive phase is photoperiod [3]. In Arabidopsis, day length-dependent regulation of the floral promoter CONSTNAS (CO) plays a central role in seasonal flowering [1]. CO gene expression levels are relatively high under light in long days, but are very low during the daytime in short days [4]. This

\footnotetext{
*Correspondence: jchong@gnu.ac.kr; younghsong@ajou.ac.kr ${ }^{1}$ Division of Life Science, Applied Life Science (BK21 Plus Program), Plant Molecular Biology and Biotechnology Research Center, Gyeongsang National University, Jinju, Gyeongnam 52828, South Korea

${ }^{3}$ Department of Life Sciences, Ajou University, Suwon, Gyeonggi 16499, South Korea

Full list of author information is available at the end of the article
}

differential diel expression profile of $C O$ is known to be the day length measurement mechanism [5, 6]. Additionally, post-translational regulation of $\mathrm{CO}$ by the function of photoreceptors is essential for day length-dependent induction of the FLOWERING LOCUS T (FT) gene, which accelerates flowering [7-10].

$\mathrm{CO}$ protein is the primary transcriptional activator of the FT gene, which encodes the mobile florigenic signal that is synthesized in the leaf phloem tissues and transmitted to the shoot apical meristem [11-14]. In long days, $\mathrm{CO}$ directly binds to the promoter region of the $F T$ gene and activates its transcription $[8,15,16]$. $\mathrm{CO}$ appears to be recruited to $\mathrm{CO}$-responsive elements (COREs) in the FT promoter through its C-terminal CCT (CONSTANS, CONSTANS-like, and TOC1) motif [15]. However, the function of $\mathrm{CO}$ in the induction of $F T$ expression largely depends on other DNA-binding transcription factors [2,17-21]. The CCAAT-box-binding 
nuclear factor $\mathrm{Y}$ (NF-Y) proteins form a trimeric complex with $\mathrm{CO}$ and affect its binding to the CORE element of the $F T$ promoter [21]. This NF-Y-CO complex regulates the dynamic changes in the structure of the $F T$ promoter region that occur throughout the day in long days by binding to a CCAAT box in the enhancer region $[2,18]$. The myb-like helix turn helix $(\mathrm{HTH})$ transcription factor family protein ASYMMETRIC LEAVES 1 (AS1) physically interacts with $\mathrm{CO}$ and is involved in photoperiodic flowering by contributing to $\mathrm{CO}$-dependent $F T$ induction in long days [20]. In contrast to NF-Y and AS1, TARGET OF EAT (TOE) proteins bind to CO and inhibit its activity [22]. In addition, the bZIP transcription factor TGACG (TGA) MOTIF-BINDING FACTOR 4/ocs element binding factor 4 (TGA4/OBF4) directly binds to the $F T$ promoter interacts with $\mathrm{CO}$ in yeast and in vitro, although the biological relevance of this interaction is not fully understood [23]. Hence, the heterodimeric complexes formed between $\mathrm{CO}$ and DNA-binding transcription factors are a crucial component of photoperiodic flowering by regulating $F T$ expression.

In this study, we report the identification of another CO-interacting protein, FLOWERING HTH 1 (FHTH1). A complex containing $\mathrm{CO}$ and FHTH1 was observed in the nucleus. Moreover, overexpression of a chimeric repression motif-fused FHTH1 significantly delayed flowering in long days. Our results suggest that FHTH1 may positively regulate flowering by binding to $\mathrm{CO}$.

\section{Materials and methods}

\section{Plant materials and growth conditions}

All Arabidopsis thaliana plants used in this study, including wild type, fhth1 (SALK_005547), fd-4 (SALK_11848C), and 35S:FHTH1-SRDX \#3, are Columbia (Col-0) ecotype. The fhth1 mutant used in this study was obtained from ABRC and was identified using the LBb1 primer (SALK) to verify the T-DNA insertion and FHTH1 gene-specific primers. For flowering experiments, all plants were grown on soil in plant incubators at $22{ }^{\circ} \mathrm{C}$ under cool white fluorescent lights in long days (16-h light/8-h dark). Flowering time was measured by recording the number of rosette leaves when plants were bolted.

\section{Yeast two-hybrid analysis}

For the yeast two-hybrid screening, the $C O$ cDNA encoding a deletion derivative of $\mathrm{CO}$ (designated $\mathrm{CO} \Delta 133-174$, with a deletion of amino acids 133-174) was constructed by overlap extension polymerase chain reaction (OEPCR) and was introduced into pDEST32 to yield the bait construct BD-CO $\Delta 133-174$. After transforming the construct into the yeast strain PJ69-4A, yeast cells harboring the $\mathrm{BD}-\mathrm{CO} \Delta 133-174$ construct were grown on SD agar medium lacking leucine (Leu) and histidine (His) with various concentrations of 3-amino-1,2,4-triazole (3-AT; $0.1,0.5,1$, and $5 \mathrm{mM}$ ) or lacking Leu, His, and Adenine (Ade) to prove that the construct is a suitable candidate as a bait. The yeast two-hybrid screening and assay procedures were described previously [20, 23]. Briefly, an Arabidopsis transcription factor (TF) library carrying about 1400 TF cDNAs in the pDEST22 AD vector was introduced into yeast cells carrying the BD-CO $\Delta 133-174$ construct. Then, the transformants were selected on SD agar medium lacking tryptophan (Trp), Leu, His, and Ade. After removing false positives, one clone, containing FHTH1 cDNA, was obtained. To validate the interaction, bait and prey vectors harboring the $\mathrm{CO} \Delta 133-174$ and FHTH1 cDNAs, respectively, were co-transformed into pJ69-4A. The transformants containing the vectors were selected on SD agar medium lacking Trp, Leu, His, and Ade.

\section{Histochemical GUS assay}

For the FHTH1:GUS reporter construct, the $5^{\prime}$ upstream sequence of the FHTH1 gene ( -2009 to -9 ; the translation initiation site was counted as +1 ) was amplified from wild-type genomic DNA and introduced into the pMDC163 vector [24]. The procedures used for plant growth and GUS staining were described previously [23].

\section{Intracellular distribution and BiFC experiments}

The tobacco (Nicotiana benthamiana) transient expression system was used for the subcellular localization and bimolecular fluorescence complementation (BiFC) assays. For subcellular localization experiments, FHTH1 and $C O$ cDNAs lacking a stop codon were amplified by PCR and introduced into the pMDC43 vector to generate $\mathrm{C}$-terminal GFP fusions [24]. The pMDC43 plasmid carrying either the 35S:FHTH1-GFP or 35S:CO-GFP construct was introduced into agrobacterium GV3101, and agrobacteria cultured overnight were individually infiltrated into 3-week-old tobacco leaves. For BiFC assay, the FHTH1 and CO cDNAs were cloned into the pDESTVYNE(R)GW and pDEST-VYCE(R)GW vectors [25], respectively, to generate $\mathrm{N}$ - and $\mathrm{C}$-terminal fusions to enhanced yellow fluorescent protein (eYFP) or Venus, generating VN-FHTH1 and VC-CO, respectively.

Agrobacteria carrying either the $35 S: V N-F H T H 1$ or 35S:VC-CO construct were co-infiltrated into tobacco leaves. GFP and YFP were visualized by a fluorescence microscope (AX70, Olympus). 


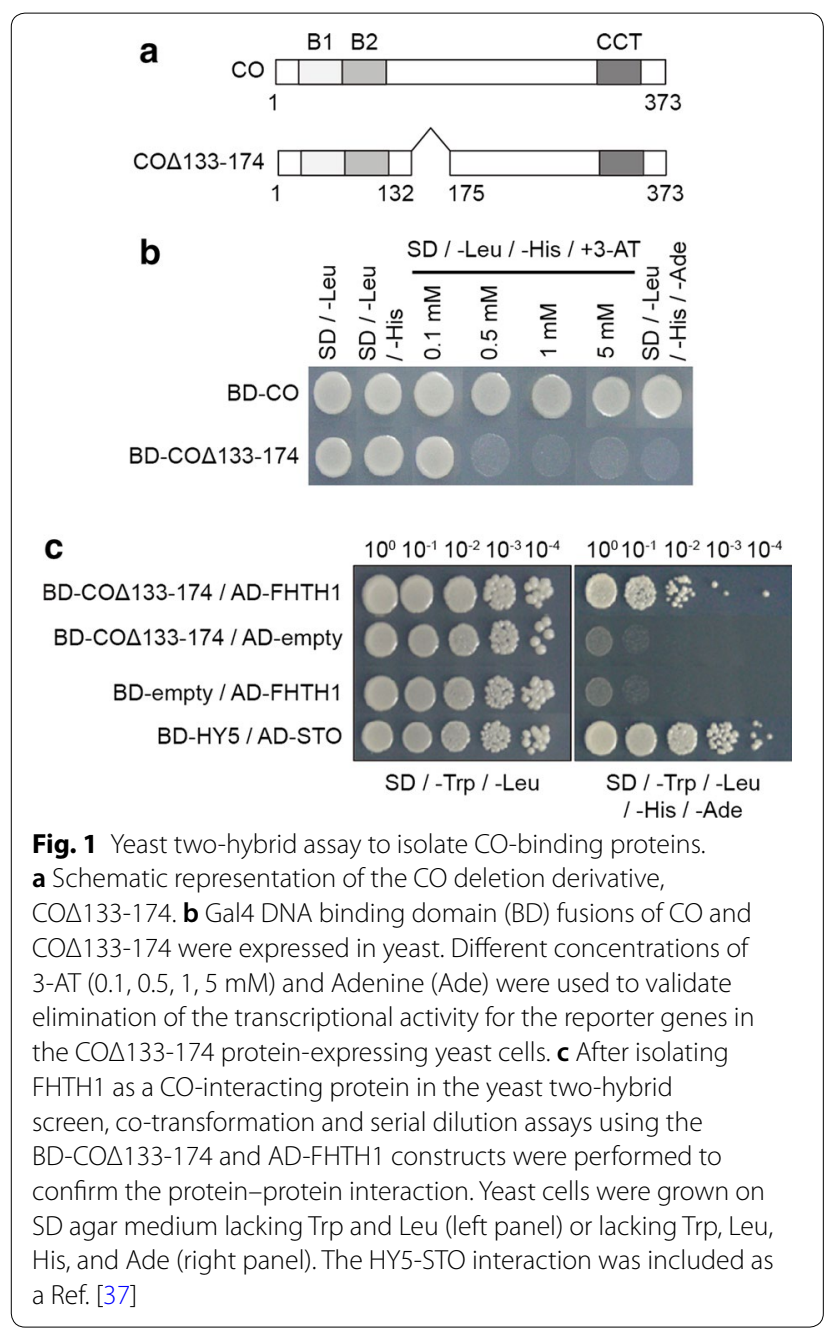

\section{Generation of transgenic plants}

To generate 35S:FHTH1-SRDX transgenic plants, the FHTH1 cDNA lacking a stop codon was introduced into the pH35GEAR vector [26]. The pH35GEAR vector carrying the 35S:FHTH1:SRDX construct was transformed into wild-type plants.

\section{Results and discussion}

\section{Isolation of $\mathrm{FHTH} 1$ as a CO-interacting protein}

$\mathrm{CO}$ is the main floral promoter in Arabidopsis under long-day conditions [27]. Although many proteins that form heterodimer complexes with $\mathrm{CO}$ have been identified $[8-10,16,17,19,20,22,23,28-30]$, the molecular mechanisms by which the transcriptional activity of $\mathrm{CO}$ regulates day length-dependent flowering still remain to be understood. Therefore, a yeast two-hybrid screen was performed to isolate additional unidentified $\mathrm{CO}$ interacting DNA-binding proteins. First, we constructed a deletion derivative of the $\mathrm{CO}$ gene that lacks the coding sequence for the glutamine-rich region (amino acids 133-174) to eliminate its intrinsic transcriptional activation potential (Fig. 1a) [15]. As expected, the mutant protein $(\mathrm{CO} \Delta 133-174)$ expressed from the deletion construct showed no self-activation on the reporter genes, which encode proteins required for the biosynthesis of histidine and adenine (Fig. 1b). Next, we introduced an Arabidopsis transcription factor library into yeast cells expressing Gal4 DNA-binding domain (BD)-CO $\Delta 133-174$, the bait peptide. One clone containing a cDNA encoding a DNA-binding transcription factor was obtained as a potential interactor with $\mathrm{CO} \Delta 133-174$, which was confirmed by co-transformation with the bait and prey vectors and subsequent serial dilution experiments (Fig. 1c). The encoded transcription factor (At5g06800) belongs to the myb-like helix turn helix $(\mathrm{HTH})$ transcription factor family and has not been previously characterized. As this gene encodes a HTH protein that regulates the timing of floral induction (as shown later), we named it FLOWERING HTH 1 (FHTH1). Our data suggests that FHTH1 can physically bind to $\mathrm{CO}$ not only in yeast but also in planta.

\section{Complex formation between $\mathrm{CO}$ and $\mathrm{FHTH} 1$ in planta}

The nuclear B-box protein $\mathrm{CO}$ regulates the expression of its target gene in response to long days [8]. Based on the amino acid sequence of its myb-like HTH domain, FHTH1 was predicted to be a DNA-binding protein. To determine the intracellular localization of FHTH1, we expressed the protein as an $\mathrm{N}$-terminal green fluorescent protein (GFP) fusion under the control of the cauliflower mosaic virus (CaMV) $35 \mathrm{~S}$ promoter in Nicotiana benthamiana leaves. GFP-FHTH1 fluorescence was observed in the nucleus of tobacco epidermal cells, resembling the localization of $\mathrm{CO}$ (Fig. 2a). This finding suggests that FHTH1 forms a protein complex with $\mathrm{CO}$ in the same subcellular localization. We then investigated where the FHTH1-CO interaction occurs in plant cells using BiFC. The FHTH1 and $\mathrm{CO}$ proteins were respectively tagged with the $\mathrm{N}$ - and $\mathrm{C}$-terminus of enhanced yellow fluorescent protein (eYFP, also called Venus) and co-expressed in tobacco leaves. A strong signal for reconstituted eYFP fluorescence, which requires the association of the $\mathrm{N}$ - and $\mathrm{C}$-termini, was only detected in the nucleus (Fig. 2b), confirming the interaction observed in yeast (Fig. 1c). These results indicate that FHTH1 and CO colocalize in the nucleus and form a complex (Fig. 2b). The $C O$ gene is expressed in the vasculature, especially phloem tissues [31, 32]. As FHTH1 physically interacts with $\mathrm{CO}$ in planta (Fig. 2b), the tissue-specific expression of the FHTH1 gene in leaves might overlap with that of $C O$. Accordingly, an expression cassette bearing the GUS marker gene under the control of a $2.0 \mathrm{~kb}$ FHTH1 


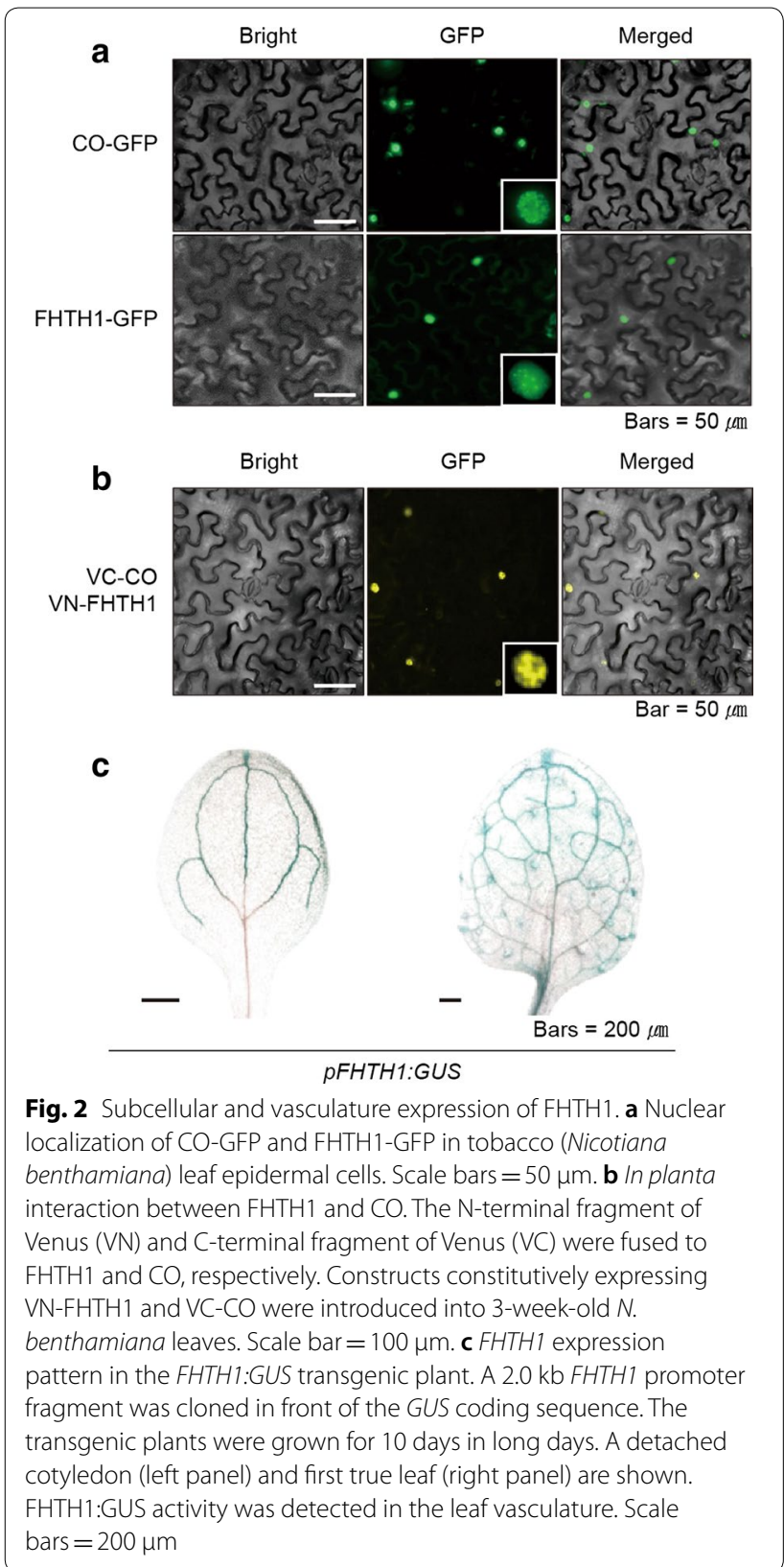

promoter was constructed and introduced into wild-type Arabidopsis plants to analyze the spatial expression pattern of FHTH1. FHTH1:GUS activity was observed in the vascular tissues of cotyledon and rosette leaves (Fig. 2c), where $\mathrm{CO}$ mainly exists, supporting the notion that FHTH1 regulates flowering through complex formation with $\mathrm{CO}$ in the phloem.

\section{FHTH1 is involved in the regulation of flowering}

Since CO functions as a floral promoter in long days, we examined whether FHTH1 regulates flowering. First, an

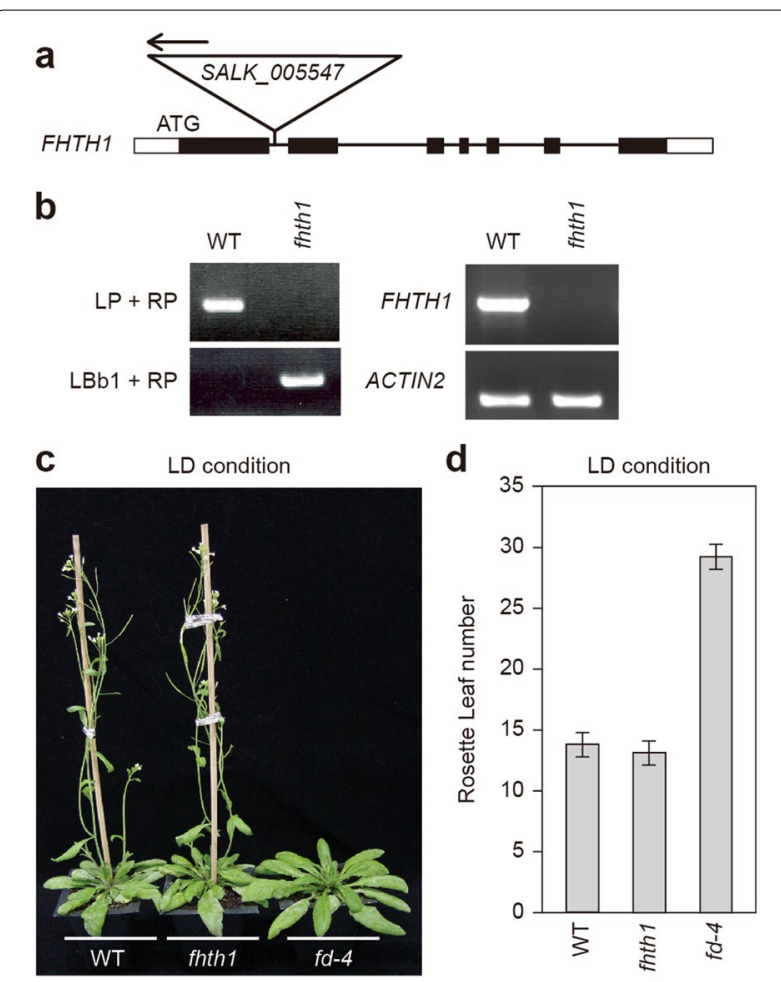

Fig. 3 Identification and flowering time of the fhth 1 mutant. a The position of the T-DNA insertion in the FHTH1 locus. The T-DNA was located in the first intron of the FHTH1 gene. Black boxes, lines, and white boxes represent exons, introns, and UTRs, respectively. b Genomic PCR (left panel) and RT-PCR (right panel) to identify the fhth 1 mutant. We characterized the homozygous mutant using combinations of the FHTH1 left primer (LP), right primer (RP), and T-DNA-specific left board primer (LBb1) for genomic PCR and comparing FHTH1 expression levels between wild-type and mutant plants. ACTIN2 was used as an internal control. c, d Flowering time of fhth 1 plants grown in long days. $d$ The number of rosette leaves were counted on the main stem. Mean \pm SD

FHTH1 T-DNA insertion mutant (SALK_005547) was obtained from public collections, and the homozygosity of $f h t h 1$ was verified by genomic PCR and RT-PCR analysis (Fig. 3a, b). We then analyzed the effect of the fhth1 mutation on flowering regulation in long days and found that the time to flowering in the mutant plants was similar to that in wild-type plants (Fig. 3c, d). This suggests that FHTH1 functions redundantly with other proteins in the same flowering pathway. To overcome this potential genetic redundancy, we generated a dominant-negative version of FHTH1 that could repress the transcription of its target gene. To this end, a DNA sequence encoding the plant-specific EAR-repression domain, also called SRDX (LDLDLELRLGFA) [33], was fused in frame to the coding region of FHTH1 (Fig. 4a). The resultant FHTH1SRDX chimeric sequence was overexpressed driven by the CaMV $35 \mathrm{~S}$ promoter in wild-type plants. Gene 


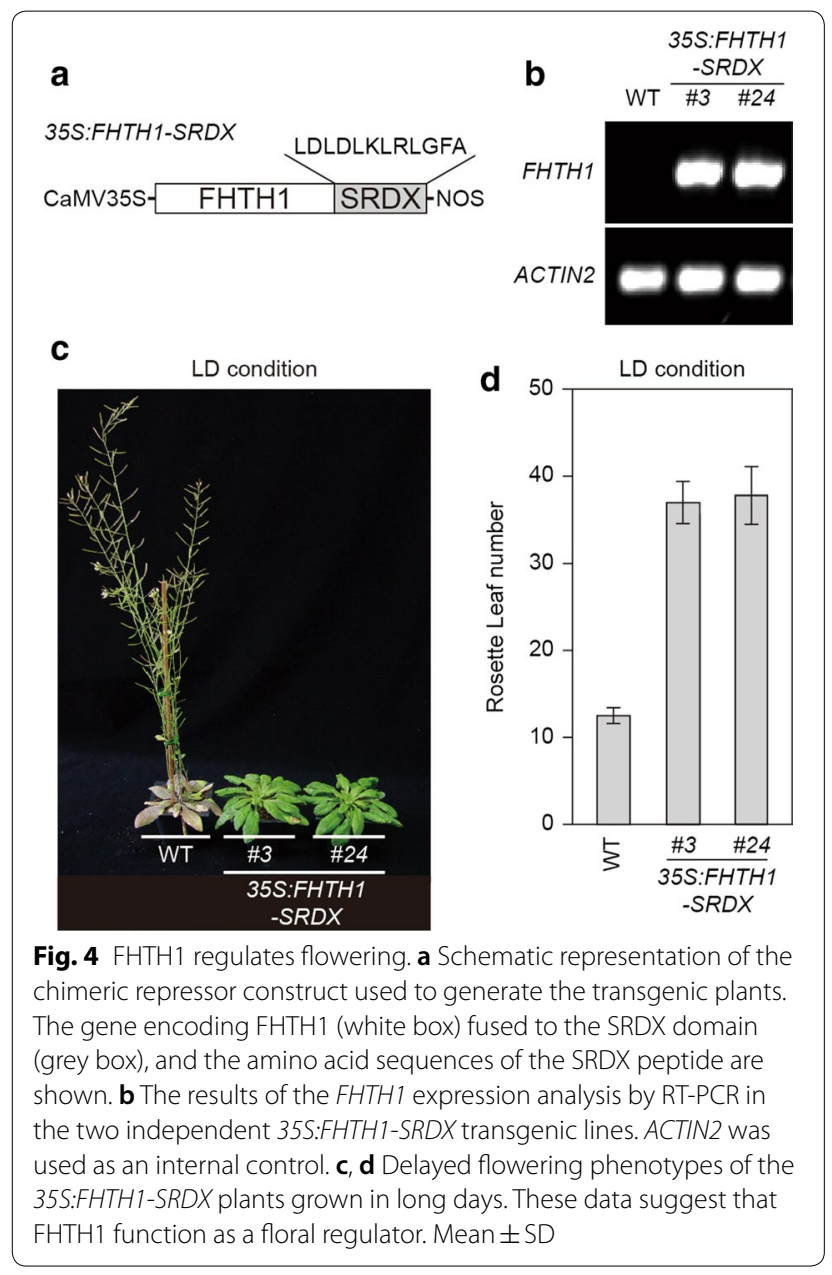

expression analysis showed high levels of FHTH1-SRDX mRNA in two independent 35S:FHTH1-SRDX lines, validating overexpression of the construct (Fig. 4b). Then, we analyzed the flowering phenotype of the two FHTH1$S R D X$ overexpressors in long days and observed a distinct late flowering phenotype (Fig. 4c, d), which resembles that of the co mutant [20], suggesting that FHTH1 likely regulates flowering time through the protein complex formation with CO.

Many plants in temperate regions have adapted the timing of their reproductive transition to the most favorable season of the year based on day length [27, 34]. In Arabidopsis thaliana, CO functions as the floral promoter by activating the expression of the florigen in response to inductive day length conditions [35]. In order to understand this photoperiod-dependent flowering, we aimed to isolate a CO-interacting transcription factor and demonstrated that FHTH1 is a new floral regulator. The formation of a nuclear complex containing FHTH1 and $\mathrm{CO}$ proteins and their overlapping tissue-specific expression (Fig. 2) imply that the two proteins function in the same flowering pathway. However, genetic analysis showed that a mutation in the FHTH1 gene had little effect on flowering time. Since transcription factors are often organized into multi-subunit complexes carrying out their biological functions [36], it is not surprising that the loss of function mutation in the FHTH1 gene did not alter the flowering phenotype (Fig. 3c, d). In contrast, the late flowering phenotype of transgenic plants overexpressing FHTH1-SRDX indicates the presence of functional redundancy between FHTH1 and other proteins in the regulation of flowering. In summary, as $\mathrm{CO}$ interacts with many DNA binding transcription factors in the regulation of its target gene [16, 17, 19, 20, 22], we suggest that FHTH1 might function in the CO-mediated flowering pathway by forming heterodimer complexes with $\mathrm{CO}$ and other $\mathrm{CO}$-interacting transcription factors. Further biochemical and genetic studies are required to understand the functional relationships among FHTH1, $\mathrm{CO}$, and other $\mathrm{CO}$-related proteins.

\section{Acknowledgements}

This project was supported by the Next-Generation BioGreen 21 Program (SSAC, Project No. PJ013386 to Y.H.S., and PJ01319801 to J.C.H, Rural Development Administration, Republic of Korea), the Basic Science Research Program funded by the Ministry of Education (NRF-2016R1D1A1B03933034 to J.C.H), NRF grant (NRF-2018R1D1A1 A09083990 to Y.H.S.), and the Nuclear R\&D Program of the Ministry of Science and ICT (MSIT, Republic of Korea) (to J.-H.K., S.S.L. and Y.H.S.). S.A.S. and S.G.W. were supported by a scholarship from the BK21 Program, Korea.

\section{Authors' contributions}

SAS and SGW performed experiments and collected data. DYH analyzed data. J-HK, SSL, and COL provided resources and interpreted data. JCH and YHS conceptualized the research, interpreted data, and wrote the manuscript. All authors read and approved the final manuscript.

\section{Funding}

This project was supported by the Next-Generation BioGreen 21 Program (SSAC, Project No. PJ013386 to Y.H.S., and PJ01319801 to J.C.H, Rural Development Administration, Republic of Korea), the Basic Science Research Program funded by the Ministry of Education (NRF-2016R1D1A1B03933034 to J.C.H), NRF grant (NRF-2018R1D1A1 A09083990 to Y.H.S.), and the Nuclear R\&D Program of the Ministry of Science and ICT (MSIT, Republic of Korea) (to J.-H.K., S.S.L. and Y.H.S.). S.A.S. and S.G.W. were supported by a scholarship from the BK21 Program, Korea.

Availability of data and materials Not applicable.

\section{Competing interests}

The authors declare that they have no competing interests.

\section{Author details}

${ }^{1}$ Division of Life Science, Applied Life Science (BK21 Plus Program), Plant Molecular Biology and Biotechnology Research Center, Gyeongsang National University, Jinju, Gyeongnam 52828, South Korea. ${ }^{2}$ Gyeongnam Agricultural Research and Extension Services, Jinju, Gyeongnam 52828, South Korea. ${ }^{3}$ Department of Life Sciences, Ajou University, Suwon, Gyeonggi 16499, South Korea. ${ }^{4}$ Advanced Radiation Technology Institute, Korea Atomic Energy Research Institute, Jeongeup, Jeonbuk 56212, South Korea. ${ }^{5}$ Department of Radiation Science and Technology, University of Science and Technology, Daejeon 34113, South Korea. ${ }^{6}$ Division of Plant Sciences, University of Missouri, Columbia, MO 65211, USA. 
Received: 30 September 2019 Accepted: 10 October 2019

Published online: 19 October 2019

\section{References}

1. Yanovsky MJ, Kay SA (2002) Molecular basis of seasonal time measurement in Arabidopsis. Nature 419(6904):308-312

2. Song YH, Shim JS, Kinmonth-Schultz HA, Imaizumi T (2015) Photoperiodic flowering: time measurement mechanisms in leaves. Annu Rev Plant Biol 66:441-464

3. Mouradov A, Cremer F, Coupland G (2002) Control of flowering time: interacting pathways as a basis for diversity. Plant Cell 14(Suppl):S111-S130

4. Suárez-López P, Wheatley K, Robson F, Onouchi H, Valverde F, Coupland G (2001) CONSTANS mediates between the circadian clock and the control of flowering in Arabidopsis. Nature 410(6832):1116-1120

5. Sawa M, Nusinow DA, Kay SA, Imaizumi T (2007) FKF1 and GIGANTEA complex formation is required for day-length measurement in Arabidopsis. Science 318(5848):261-265

6. Song YH, Ito S, Imaizumi T (2013) Flowering time regulation: photoperiod- and temperature-sensing in leaves. Trends Plant Sci 18(10):575-583

7. Valverde F, Mouradov A, Soppe W, Ravenscroft D, Samach A, Coupland G (2004) Photoreceptor regulation of CONSTANS protein in photoperiodic flowering. Science 303(5660):1003-1006

8. Song YH, Smith RW, To BJ, Millar AJ, Imaizumi T (2012) FKF1 conveys timing information for CONSTANS stabilization in photoperiodic flowering. Science 336(6084):1045-1049

9. Song YH, Estrada DA, Johnson RS, Kim SK, Lee SY, MacCoss MJ, Imaizumi $T$ (2014) Distinct roles of FKF1, GIGANTEA, and ZEITLUPE proteins in the regulation of CONSTANS stability in Arabidopsis photoperiodic flowering. Proc Natl Acad Sci USA 111(49):17672-17677

10. Lazaro A, Mouriz A, Piñeiro M, Jarillo JA (2015) Red light-mediated degradation of CONSTANS by the E3 ubiquitin ligase HOS1 regulates photoperiodic flowering in Arabidopsis. Plant Cell 27(9):2437-2454

11. Samach A, Onouchi H, Gold SE, Ditta GS, Schwarz-Sommer Z, Yanofsky MF, Coupland G (2000) Distinct roles of CONSTANS target genes in reproductive development of Arabidopsis. Science 288(5471):1613-1616

12. Corbesier L, Vincent C, Jang S, Fornara F, Fan Q, Searle l et al (2007) FT protein movement contributes to long-distance signaling in floral induction of Arabidopsis. Science 316(5827):1030-1033

13. Jaeger KE, Wigge PA (2007) FT protein acts as a long-range signal in Arabidopsis. Curr Biol 17(12):1050-1054

14. Mathieu J, Warthmann N, Küttner F, Schmid M (2007) Export of FT protein from phloem companion cells is sufficient for floral induction in Arabidopsis. Curr Biol 17(12):1055-1060

15. Tiwari SB, Shen Y, Chang H-C, Hou Y, Harris A, Ma SF, McPartland M et al (2010) The flowering time regulator CONSTANS is recruited to the FLOWERING LOCUS T promoter via a unique cis-element. New Phytol 187(1):57-66

16. Hayama R, Sarid-Krebs L, Richter R, Fernández V, Jang S, Coupland G (2017) PSEUDO RESPONSE REGULATORs stabilize CONSTANS protein to promote flowering in response to day length. EMBO J 36(7):904-918

17. Wenkel S, Turck F, Singer K, Gissot L, Le Gourrierec J, Samach A, Coupland $\mathrm{G}$ (2006) CONSTANS and the CCAAT box binding complex share a functionally important domain and interact to regulate flowering of Arabidopsis. Plant Cell 18(11):2971-2984

18. Cao S, Kumimoto RW, Gnesutta N, Calogero AM, Mantovani R, Holt BF 3rd (2014) A distal CCAAT/NUCLEAR FACTOR Y complex promotes chromatin looping at the FLOWERING LOCUST promoter and regulates the timing of flowering in Arabidopsis. Plant Cell 26(3):1009-1017

19. Kumimoto RW, Zhang Y, Siefers N, Holt BF 3rd (2010) NF-YC3, NF-YC4 and NF-YC9 are required for CONSTANS-mediated, photoperiod-dependent flowering in Arabidopsis thaliana. Plant J 63(3):379-391
20. Song YH, Lee I, Lee SY, Imaizumi T, Hong JC (2012) CONSTANS and ASYMMETRIC LEAVES 1 complex is involved in the induction of FLOWERING LOCUS T in photoperiodic flowering in Arabidopsis. Plant J 69(2):332-342

21. Gnesutta N, Kumimoto RW, Swain S, Chiara M, Siriwardana C, Horner DS, Holt BF 3rd, Mantovani R (2017) CONSTANS imparts DNA sequence specificity to the histone fold NF-YB/NF-YC dimer. Plant Cell 29(6):1516-1532

22. Zhang B, Wang L, Zeng L, Zhang C, Ma H (2015) Arabidopsis TOE proteins convey a photoperiodic signal to antagonize CONSTANS and regulate flowering time. Genes Dev 29(9):975-987

23. Song YH, Song NY, Shin SY, Kim HJ, Yun DJ, Lim CO, Lee SY, Kang KY, Hong JC (2008) Isolation of CONSTANS as a TGA4/OBF4 interacting protein. Mol Cells 25(4):559-565

24. Curtis MD, Grossniklaus U (2003) A gateway cloning vector set for high-throughput functional analysis of genes in planta. Plant Physiol 133(2):462-469

25. Gehl C, Waadt R, Kudla J, Mendel RR, Hänsch R (2009) New GATEWAY vectors for high throughput analyses of protein-protein interactions by bimolecular fluorescence complementation. Mol Plant 2(5):1051-1058

26. Kubo M, Udagawa M, Nishikubo N, Horiguchi G, Yamaguchi M, Ito J, Mimura T, Fukuda H, Demura T (2005) Transcription switches for protoxylem and metaxylem vessel formation. Genes Dev 19(16):1855-1860

27. Song YH (2016) The effect of fluctuations in photoperiod and ambient temperature on the timing of flowering: time to move on natural environmental conditions. Mol Cells 39(10):715-721

28. Lazaro A, Valverde F, Piñeiro M, Jarillo JA (2012) The Arabidopsis E3 ubiquitin ligase HOS1 negatively regulates CONSTANS abundance in the photoperiodic control of flowering. Plant Cell 24(3):982-999

29. Graeff M, Straub D, Eguen T, Dolde U, Rodrigues V, Brandt R, Wenkel S (2016) MicroProtein-mediated recruitment of CONSTANS into a TOPLESS trimeric complex represses flowering in Arabidopsis. PLoS Genet 12(3):e1005959

30. Jing Y, Guo Q, Zha P, Lin R (2019) The chromatin-remodelling factor PICKLE interacts with CONSTANS to promote flowering in Arabidopsis. Plant Cell Environ 42(8):2495-2507

31. Takada S, Goto K (2003) TERMINAL FLOWER2, an Arabidopsis homolog of HETEROCHROMATIN PROTEIN1, counteracts the activation of FLOWERING LOCUS T by CONSTANS in the vascular tissues of leaves to regulate flowering time. Plant Cell 15(12):2856-2865

32. An H, Roussot C, Suárez-López P, Corbesier L, Vincent C, Piñeiro M et al (2004) CONSTANS acts in the phloem to regulate a systemic signal that induces photoperiodic flowering of Arabidopsis. Development 131(15):3615-3626

33. Hiratsu K, Matsui K, Koyama T, Ohme-Takagi M (2003) Dominant repression of target genes by chimeric repressors that include the EAR motif, a repression domain, in Arabidopsis. Plant J 34(5):733-739

34. Andrés F, Coupland $G$ (2012) The genetic basis of flowering responses to seasonal cues. Nat Rev Genet 13(9):627-639

35. Song YH, Kubota A, Kwon MS, Covington MF, Lee N, Taagen ER et al (2018) Molecular basis of flowering under natural long-day conditions in Arabidopsis. Nat Plants 4(10):824-835

36. Ito S, Song YH, Josephson-Day AR, Miller RJ, Breton G, Olmstead RG, Imaizumi T (2012) FLOWERING BHLH transcriptional activators control expression of the photoperiodic flowering regulator CONSTANS in Arabidopsis. Proc Natl Acad Sci USA 109(9):3582-3587

37. Jiang L, Wang Y, Li QF, Björn LO, He JX, Li SS (2012) Arabidopsis STO/ BBX24 negatively regulates UV-B signaling by interacting with COP1 and repressing HY5 transcriptional activity. Cell Res 22(6):1046-1057

\section{Publisher's Note}

Springer Nature remains neutral with regard to jurisdictional claims in published maps and institutional affiliations. 